\title{
IMPROVED ANALYSIS OF A PROPPED CANTILEVER UNDER LATERAL VIBRATION
}

\author{
Victor O. Okonkwo ${ }^{1}$, Chukwurah H. Aginam ${ }^{1}$ and Charles M. O. Nwaiwu' \\ Nnamdi Azikiwe University, Faculty of Engineering, Department of Civil Engineering, \\ P.M.B.1105 Awka, Nigeria;vo.okonkwo@unizik.edu.ng
}

\begin{abstract}
Continuous systems can be analysed as lumped masses connected by massless elements. This reduces the structure's degree of freedom and therefore simplifies the analysis. However, this over simplification introduces an error in the analysis and the results are therefore approximate. In this work, sections of the vibrating beam were isolated and the equations of the forces causing vibration obtained using the Hamilton's principle. These forces were applied to the nodes of an equivalent lumped mass beam and the stiffness modification needed for it to behave as a continuous beam obtained. The beam's stiffness was modified using a set of stiffness modification factors $\phi_{1}$ to $\phi_{4}$. It was observed that by applying these factors in the dynamic analysis of the beam using the Lagrange's equation, we obtain the exact values of the fundamental frequency irrespective of the way the mass of the beam was lumped. From this work we observed that in order to obtain an accurate dynamic response from a lumped mass beam there is need to modify the stiffness composition of the system and no linear modification of the stiffness distribution of lumped mass beams can cause them to be dynamically equivalent to the continuous beams. This is so because the values of the modification factors obtained for each beam segment were not equal. The stiffness modification factors were obtained for elements at different sections of the beam
\end{abstract}

\section{KEYWORDS}

Lagrange equations, Stiffness matrix, Inertia matrix, Lumped mass, Natural frequency

\section{INTRODUCTION}

Most imposed loads on structures are dynamic in nature. They either vary in time or in space. The structure therefore vibrates frequently under the effect of these loads. Structural and mechanical systems in general consist of structural components which have distributed mass and elasticity [1]. All bodies possessing mass and elasticity are capable of vibration [2, 3]. The dynamic analysis of structures can be done using the Newton's equation of motion. This is possible for very simple structures with few degrees of freedom. But as the degrees of freedom increase the resulting equations become very cumbersome and an energy method is preferred [4].

The earliest energy method for such analysis is the Lagrange's equations. These equations were formulated for lumped masses connected by massless elements [5]. The masses are assumed to be concentrated at specified points known as nodes. When used to model a system with a continuous distribution of mass, this method will give an approximate result. The accuracy of the results will however increase with an increase in the number of lumped masses and uniformity in their spacing. An increase in the number of lumped mass increases the size of the resulting matrix and hence the size of the required computational analysis. A better energy method is the Hamilton's principle. This is an extension of the principle of minimum potential energy. This method enables us to formulate partial differential equations for the analysis of the structure as uniform systems (i.e. 
structures with uniformly distributed masses) $[6,7]$. The results are exact. Its major drawback is that it is very difficult to formulate the differential equations of complex structural systems using this method.

The prevalence of computers has increased the use of numerical methods in structural analysis [8]. Finite difference method, Ritz method, Rayleigh-ritz method and finally the finite element method is today widely used in such analysis. The finite element method is the most popular and like the Ritz approach incorporates the use of shape function. These shape functions are used to formulate inertia matrix known as the consistency matrix [9]. If the shape function is truly representative of the deformed shape of the structure, the consistency matrix should be equal to the equivalent mass matrix. The equivalent mass matrix for a segment of a continuous system is one that returns precisely the dynamic properties of the original segment in discretized form [10]. The power of the finite element method is further enhanced by its ability to subdivide the structure into finite elements, the smaller the size of the finite elements the better the results from their analysis. This has made it a widely applied tool in researches structural analysis [11, 12, 13]. However increasing the number of elements however increases the size of the matrices to analyze and therefore increases the computational cost.

Despite the rapid advances in these numerical approaches, the lumping of continuous masses has persisted due to its visual appeal and its simplification of the analysis. Mass lumping distorts the mass distribution and leads to a less representative inertia matrix [14]. This introduces an error in the analysis which ultimately affects the values of the computed natural frequencies of the structure. It has limited number of coordinates and may not fully account for the structural characteristics of a system accurately [15]. Despite these limitations It is still widely used in introductory topics in structural dynamics and in advanced research involving complex systems [16 - 19].

Efforts have been made in time past to generate better equivalent mass matrices for analysis of continuous systems [20,21, 22]. Also Ericson and Parker [23] suggested that varying the stiffness of the structural system would lead to better analysis results. This was implemented for longitudinally vibrating bars using a set of two stiffness modification factors [24]. In this work, the variation of the structure's stiffness distribution as a means of nullifying the effect of lumping of continuous mass in laterally vibrating beams was explored.

\section{Mathematical Theory}

The partial differential equation governing the free lateral vibration of a beam is given by [26]

$$
E I u^{I V}+\mu \ddot{u}=0
$$

Where $\mathrm{El}$ is the flexural rigidity of the beam and $\mu$ is its mass per unit length. For a harmonic vibration and by applying the boundary conditions for the beam we obtain four equations that can be solved numerically to give us the roots $\beta_{j} L$ from which the natural frequency of vibration $\omega_{j}$ is computed.

$$
\beta^{4}=\frac{\mu \omega^{2}}{E I}
$$

The mode shape is obtained as [25]

$$
\emptyset_{j}(x)=\cosh \beta_{j} x+b_{2 j} \sinh \beta_{j} x-\cos \beta_{j} x+b_{4 j} \sin \beta_{j} x
$$

Where

$$
b_{2 j}=\frac{\cos \beta_{j} L-\cosh \beta_{j} L}{\sinh \beta_{j} L-\sin \beta_{j} L}
$$




$$
b_{4 j}=\frac{\cosh \beta_{j} L-\cos \beta_{j} L}{\sinh \beta_{j} L-\sin \beta_{j} L}
$$

Equation (3) is the equation of the $\mathrm{j}^{\text {th }}$ mode of vibration of the fixed-pinned beam. The first mode of vibration $(\mathrm{j}=1)$ can be obtained by substituting $\beta_{j} L=\beta_{1} L=3.92660232$ into (3). The second mode of vibration $\left(\mathrm{j}=2\right.$ ) can be obtained by substituting $\beta_{j} L=\beta_{2} L=7.06858275$ into the equation. The general solution by mode superposition is [24][25]

$$
u_{1}\left(x_{1}, t\right)=\sum_{j=1}^{\infty} \phi_{j}\left(x_{1}\right)\left(A_{j} \cos w_{j} t+B_{j} \sin w_{j} t\right)
$$

Where the constants $A_{j}$ and $B_{j}$ can be determined from the initial conditions.

\section{METHODS}

The vibrations of structural systems are governed by two essential components; the structure's mass distribution and the structure's stiffness [24, 27]. These properties are represented by the structure's inertia matrix and stiffness matrix. If we alter the mass distribution we will expect a corresponding change in the stiffness distribution. The lumping of continuous mass at specified nodes alters the mass distribution (inertia matrix). There is need to find the corresponding modification in the stiffness distribution needed to restore the vibration characteristics of the system. This as in [24] was done by equating two equations. One is the force equilibrium equation while the other is the equation of motion of a vibrating system.

The force equilibrium equations and the equations of motion are force equations. Force equilibrium equations have been largely applied in statics [28]. Just as in [24]. It can also be applied in dynamics if the equations for the vector of fixed end moments/forces $\{F\}$ are formulated. The structure with continuous mass distribution was analyzed using the Hamilton's principle and the equations for the fixed end forces $\{F\}$ and nodal displacements $\{D\}$ formulated for any arbitrary segment of a laterally vibrating beam at time $t=0$. These were used to get the vector of nodal forces $\{P\}$ causing the vibration.

The equations of motion were used to simulate the lumped mass beam. For a vibrating element of the real beam (beam with continuous mass) and that of a corresponding element of a lumped mass beam to be equivalent then their nodal deformation $\{D\}$ and forces acting on their nodes $\{P\}$ must be equal [24].

Figure 1 shows a propped cantilever beam under inertia forces. A segment of the beam shown is being restrained by the fixed end forces $F_{1}$ to $F_{4}$.

From the D'Alembert principle the forces on the vibrating beam can be calculated from its inertia force [29]. For an elementary part of the beam at a distance $z$ from the origin this force is $\mu \ddot{u} d z$. (See Figure 1) 


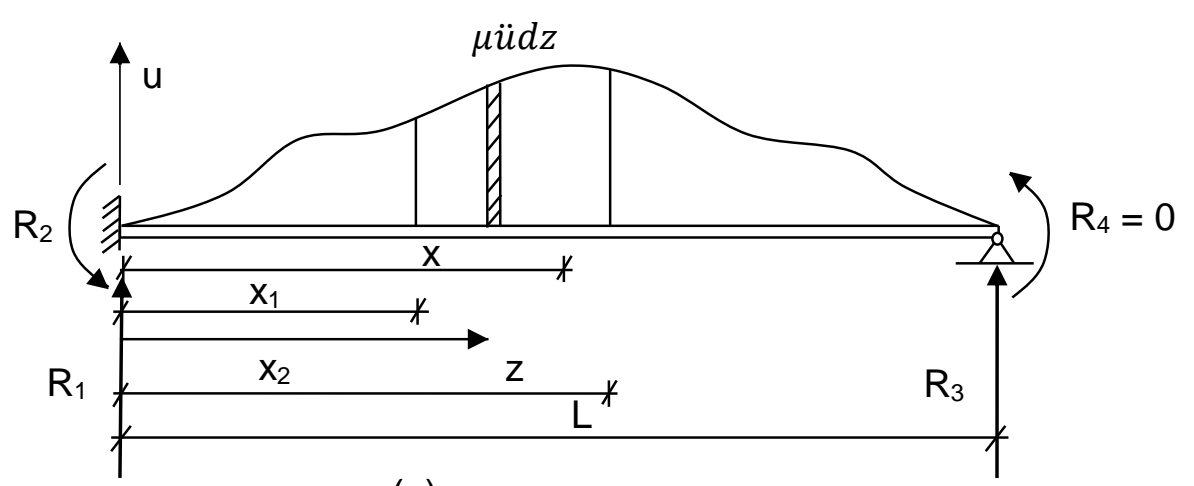

(a)

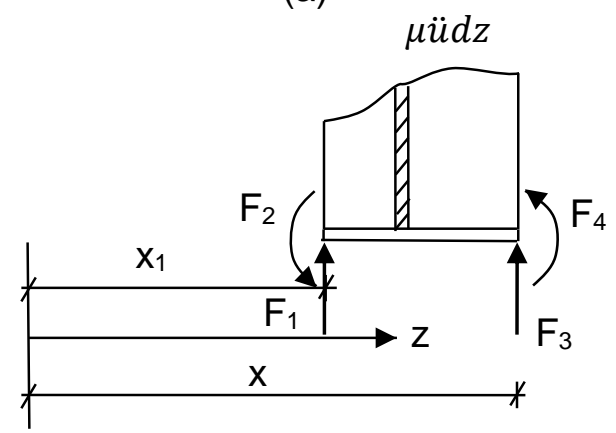

(b)

Fig. 1 - (a) A fixed-pinned beam under lateral vibration due to the inertial forces $\mu \ddot{u}$ forces $\mu \ddot{u}$

(b) A segment of the beam under longitudinal vibration due to inertial

Using the principle of virtual work and the flexibility method we determine the fixed end forces $F_{1}$ to $F_{4}$ of the isolated element of the excited beam of Figure $1 b$ to be

$$
\begin{gathered}
F_{1}=-6 \sum_{j=1}^{\infty} \frac{E I A_{j}}{L^{3}\left(\xi_{2}-\xi_{1}\right)^{3}} W_{1} \\
F_{2}=-2 \sum_{j=1}^{\infty} \frac{E I A_{j}}{L^{2}\left(\xi_{2}-\xi_{1}\right)^{2}} W_{2} \\
F_{3}=\sum_{j=1}^{\infty} \frac{E I A_{j}}{L^{3}\left(\xi_{2}-\xi_{1}\right)^{3}}\left[6 W_{1}+\beta_{j}^{3} L^{3}\left(\xi_{2}-\xi_{1}\right)^{3}\left(\sinh \beta_{j} L \xi_{2}-\sinh \beta_{j} L \xi_{1}-\sin \beta_{j} L \xi_{2}+\sin \beta_{j} L \xi_{1}+\right.\right. \\
\left.\left.b_{2 j}\left(\cosh \beta_{j} L \xi_{2}-\cosh \beta_{j} L \xi_{1}\right)-b_{4 j}\left(\cos \beta_{j} L \xi_{2}-\cos \beta_{j} L \xi_{1}\right)\right)\right] \\
F_{4}=\sum_{j=1}^{\infty} \frac{E I A_{j}}{L^{2}\left(\xi_{2}-\xi_{1}\right)^{2}}\left[-6 W_{1}+2 W_{2}-\beta_{j}^{3} L^{3}\left(\xi_{2}-\xi_{1}\right)^{3}\left(-\sinh \beta_{j} L \xi_{1}-b_{2 j} \cosh \beta_{j} L \xi_{1}+\sin \beta_{j} L \xi_{1}+\right.\right. \\
\left.b_{4 j} \cos \beta_{j} L \xi_{1}\right)+\left(\cosh \beta_{j} L \xi_{2}+b_{2 j} \sinh \beta_{j} L \xi_{2}+\cos \beta_{j} L \xi_{2}-b_{4 j} \sin \beta_{j} L \xi_{2}\right)-\left(\cosh \beta_{j} L \xi_{1}+\right. \\
\left.\left.b_{2 j} \sinh \beta_{j} L \xi_{1}+\cos \beta_{j} L \xi_{1}-b_{4 j} \sin \beta_{j} L \xi_{1}\right)\right]
\end{gathered}
$$

Where

$$
\begin{array}{r}
W_{1}=\beta_{j}^{3} L^{3}\left(\frac{\left(\xi_{2}+\xi_{1}\right)\left(\xi_{2}^{2}-\xi_{1}^{2}\right)}{2}-\frac{2\left(\xi_{2}^{3}-\xi_{1}^{3}\right)}{3}\right)\left(-\sinh \beta_{j} L \xi_{1}-b_{2 j} \cosh \beta_{j} L \xi_{1}+\sin \beta_{j} L \xi_{1}+b_{4 j} \cos \beta_{j} L \xi_{1}\right)+ \\
b_{2 j}\left(\beta L\left(\xi_{1}-\xi_{2}\right) \cosh \beta_{j} L \xi_{2}+2 \sinh \beta_{j} L \xi_{2}-\beta_{j} L\left(\xi_{2}-\xi_{1}\right) \cosh \beta_{j} L \xi_{1}-2 \sinh \beta_{j} L \xi_{1}\right)-
\end{array}
$$




$$
\begin{array}{r}
b_{4 j}\left(-\beta_{j} L\left(\xi_{1}-\xi_{2}\right) \cos \beta_{j} L \xi_{2}-2 \sin \beta_{j} L \xi_{2}+\beta_{j} L\left(\xi_{2}-\xi_{1}\right) \cos \beta_{j} L \xi_{1}+2 \sin \beta_{j} L \xi_{1}\right)+\beta_{j} L\left(\xi_{1}-\right. \\
\left.\xi_{2}\right) \sinh \beta_{j} L \xi_{2}-\beta_{j} L\left(\xi_{2}-\xi_{1}\right) \sinh \beta_{j} L \xi_{1}+\beta_{j} L\left(\xi_{1}-\xi_{2}\right) \sin \beta_{j} L \xi_{2}-\beta_{j} L\left(\xi_{2}-\xi_{1}\right) \sin \beta_{j} L \xi_{1}+ \\
2 \cosh \beta_{j} L \xi_{2}-2 \cosh \beta_{j} L \xi_{1}-2 \cos \beta_{j} L \xi_{2}+2 \cos \beta_{j} L \xi_{1} \\
W_{2}=\beta_{j}^{3} L^{3}\left(\frac{\left(2 \xi_{2}+\xi_{1}\right)\left(\xi_{2}^{2}-\xi_{1}^{2}\right)}{2}-\frac{\xi_{1}\left(\xi_{2}-\xi_{1}\right)^{2}}{2}-\left(\xi_{2}^{3}-\xi_{1}^{3}\right)\right)\left(-\sinh \beta_{j} L \xi_{1}-b_{2 j} \cosh \beta_{j} L \xi_{1}+\sin \beta_{j} L \xi_{1}+\right. \\
\left.b_{4 j} \cos \beta_{j} L \xi_{1}\right)-\left(\frac{\beta_{j}^{2} L^{2}\left(\xi_{2}-\xi_{1}\right)^{2}}{2}\right)\left(\cosh \beta_{j} L \xi_{1}+b_{2 j} \sinh \beta_{j} L \xi_{1}+\cos \beta_{j} L \xi_{1}-b_{4 j} \sin \beta_{j} L \xi_{1}\right)+ \\
\beta_{j} L\left(\xi_{1}-\xi_{2}\right) \sinh \beta_{j} L \xi_{2}-2 \beta_{j} L\left(\xi_{2}-\xi_{1}\right) \sinh \beta_{j} L \xi_{1}+\beta_{j} L\left(\xi_{1}-\xi_{2}\right) \sin \beta_{j} L \xi_{2}-2 \beta_{j} L\left(\xi_{2}-\xi_{1}\right) \sin \beta_{j} L \xi_{1}- \\
3 \cos \beta_{j} L \xi_{2}+3 \cos \beta_{j} L \xi_{1}+3 \cosh \beta_{j} L \xi_{2}-3 \cosh \beta_{j} L \xi_{1}+b_{2 j}\left(\beta_{j} L\left(\xi_{1}-\xi_{2}\right) \cosh \beta_{j} L \xi_{2}-\right. \\
\left.2 \beta_{j} L\left(\xi_{2}-\xi_{1}\right) \cosh \beta_{j} L \xi_{1}+3 \sinh \beta_{j} L \xi_{2}-3 \sinh \beta_{j} L \xi_{1}\right)-b_{4 j}\left(-\beta_{j} L\left(\xi_{1}-\xi_{2}\right) \cos \beta_{j} L \xi_{2}+\right. \\
\left.2 \beta_{j} L\left(\xi_{2}-\xi_{1}\right) \cos \beta_{j} L \xi_{1}-3 \sin \beta_{j} L \xi_{2}+3 \sin \beta_{j} L \xi_{1}\right) \\
\xi_{1}=x_{1} /{ }_{L}, \xi_{2}=x_{2} / L
\end{array}
$$

For us to be able to evaluate these equations (for the fixed end forces $F_{1}, F_{2}, F_{3}$ and $F_{4}$ ) there is need to derive an expression for $A_{j}$ for a fixed-pinned beam.

Consider a uniform propped cantilever beam under the action of its self weight as shown in Figure 2. $\mu$ is the mass per unit length of the beam and $g$ is the acceleration due to gravity.

From the equation of elastic curve and by considering the initial boundary conditions and the equation for the static deflection of the uniform beam under its self weight, let the initial deflection of the beam (at time $\mathrm{t}=0$ ) be

$$
u(x, 0)=b L\left(\frac{5 x^{3}}{L^{3}}-\frac{2 x^{4}}{L^{4}}-\frac{3 x^{2}}{L^{2}}\right)
$$

where $b$ is a dimensionless constant equal to $\frac{\mu g L^{3}}{48 E I}$.

Then from [19] by substituting equations (3) and (13)

$$
\begin{gathered}
A_{j}=\frac{\mu}{M_{j}} \int_{0}^{L} u(x, 0) \emptyset_{j} d x=\frac{b \mu L^{2}}{M_{j}}\left[\frac{-\left(\cosh \beta_{j} L+\cos \beta L\right)}{\beta_{j}^{2} L^{2}}+\frac{18\left(\cosh \beta_{j} L-\cos \beta_{j} L\right)}{\beta_{j}^{4} L^{4}}-\frac{48\left(\sinh \beta_{j} L-\sin \beta_{j} L\right)}{\beta_{j}^{5} L^{5}}+\right. \\
\left.b_{2 j}\left(\frac{-2\left(\sinh \beta_{j} L+\sin \beta_{j} L\right)}{\beta_{j}^{2} L^{2}}+\frac{18\left(\sinh \beta_{j} L-\sin \beta_{j} L\right)}{\beta_{j}^{4} L^{4}}-\frac{48\left(\cosh \beta_{j} L+\cos \beta_{j} L-2\right)}{\beta_{j}^{5} L^{5}}\right)\right]
\end{gathered}
$$

Equation (14) is an expression for the constant $A_{j}$ for a propped cantilever beam under an initial lateral displacement caused by its self weight. It can be substituted into Equations (7) to (10) to obtain the values of the fixed end forces $F_{1}, F_{2}, F_{3}$ and $F_{4}$. With these equations the force equilibrium equations for segments of a vibrating beam can be written and the inherent nodal forces in the system that is causing motion calculated. An arbitrary segment of a vibrating element is identified by means of the normalized distances $\xi_{1}$ and $\xi_{2}$ of its nodes from an origin. Having obtained the fixed end forces, the vector of nodal forces $\{P\}$ is obtained from the force equilibrium. 


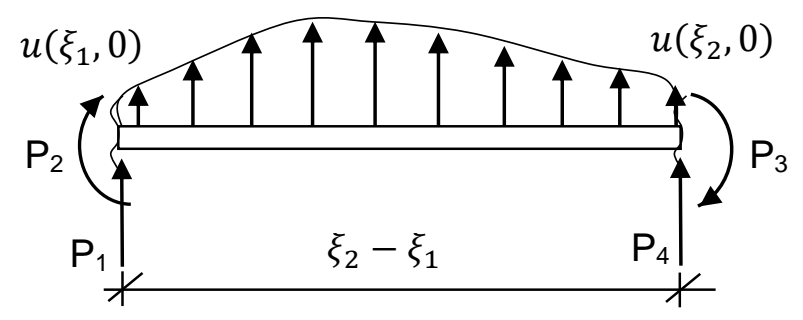

(a)

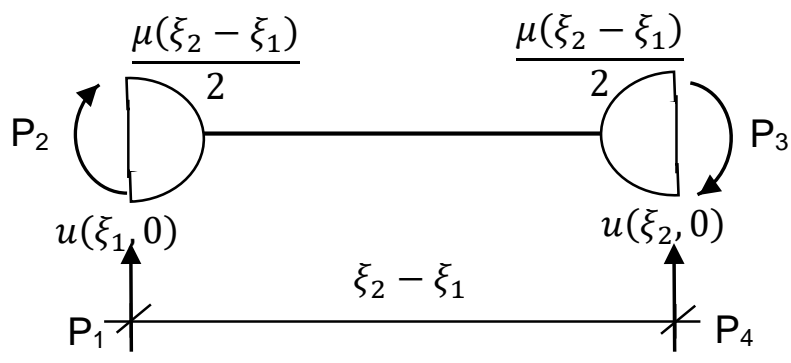

(b)

Fig. 3 - (a)An isolated segment of the laterally vibrating continuous beam showing the nodal forces $P_{1}, P_{2}, P_{3}$ and $P_{4}$

(b) An equivalent lumped mass segment showing the nodal forces

If a segment of a vibrating beam is isolated it will be in equilibrium with the application of the force vector $\{P\}$ (see Figure 3 ). The force vector $\{P\}$ represents the effect of the removed adjourning elements on the isolated segment. When the continuous bar is represented by a lumped mass bar just like the real segment the equivalent segment is supported by the same nodal forces $P_{1}, P_{2} P_{3}$ and $\mathrm{P}_{4}$ and has the same nodal displacements as the continuous/real bar.

The equation of motion for the lumped mass vibrating beam is taken as [24]

$$
[m]\{\ddot{u}\}+\left[k_{d}\right]\{u\}=\{P\}
$$

Where $[\mathrm{m}]$ is the inertial matrix, $\{u\}$ is a vector of nodal displacement and $k_{d}$ is the stiffness of the lumped mass segment under consideration. The proposed stiffness matrix for the lumped mass segment $k_{d}$ is written as

$$
\left[k_{d}\right]=\left[\begin{array}{cccc}
\frac{12 E I}{l^{3}} \phi_{1} & \frac{6 E I}{l^{2}} \phi_{2} & -\frac{12 E I}{l^{3}} \phi_{3} & \frac{6 E I}{l^{2}} \phi_{4} \\
\frac{6 E I}{l^{2}} \phi_{2} & \frac{4 E I}{l} \phi_{1} & -\frac{6 E I}{l^{2}} \phi_{4} & \frac{2 E I}{l} \phi_{3} \\
-\frac{12 E I}{l^{3}} \phi_{3} & -\frac{6 E I}{l^{2}} \phi_{4} & \frac{12 E I}{l^{3}} \phi_{1} & -\frac{6 E I}{l^{2}} \phi_{2} \\
\frac{6 E I}{l^{2}} \phi_{4} & \frac{2 E I}{l} \phi_{3} & -\frac{6 E I}{l^{2}} \phi_{2} & \frac{4 E I}{l} \phi_{1}
\end{array}\right]
$$

Where $\phi_{1}, \phi_{2}, \phi_{3}$ and $\phi_{4}$ are the stiffness modification factors for lateral vibration. They are to help redistribute the stiffness of the lumped mass segment in such a way as to annul the effect of the discretization of the beam mass due to the lumping of its distributed mass on selected nodes.

By rearranging (15) we obtain 


$$
\left\{\begin{array}{l}
\phi_{1} \\
\phi_{2} \\
\phi_{3} \\
\phi_{4}
\end{array}\right\}=\left[\begin{array}{cccc}
\frac{12 E I}{l^{3}} u_{11} & \frac{6 E I}{l^{2}} u_{21} & -\frac{12 E I}{l^{3}} u_{31} & \frac{6 E I}{l^{2}} u_{41} \\
\frac{4 E I}{l} u_{21} & \frac{6 E I}{l^{2}} u_{11} & \frac{2 E I}{l} u_{41} & -\frac{6 E I}{l^{2}} u_{3} \\
\frac{12 E I}{l^{3}} u_{31} & -\frac{6 E I}{l^{2}} u_{41} & -\frac{12 E I}{l^{3}} u_{11} & -\frac{6 E I}{l^{2}} u_{21} \\
\frac{4 E I}{l} u_{41} & -\frac{6 E I}{l^{2}} u_{31} & \frac{2 E I}{l} u_{21} & \frac{6 E I}{l^{2}} u_{11}
\end{array}\right]^{-1}\left\{\begin{array}{c}
P_{1}+\frac{\mu\left(\xi_{2}-\xi_{1}\right)}{2} \omega^{2} u_{11} \\
P_{2} \\
P_{3}+\frac{\mu\left(\xi_{2}-\xi_{1}\right)}{2} \omega^{2} u_{31} \\
P_{4}
\end{array}\right\}
$$

Equation (17) is a mathematical expression for calculating the four stiffness modification factors $\phi_{1}, \phi_{2}, \phi_{3}$ and $\phi_{4}$ for a segment of a beam under lateral vibration. $\mu$ is the mass per unit length of the beam. $\omega$ is the fundamental frequency of the vibrating mass, $u_{11}, u_{21}, u_{31}, u_{41}$ are the values of nodal displacements $\mathrm{u}_{1}, \mathrm{u}_{2}, \mathrm{u}_{3}$ and $\mathrm{u}_{4}$ for the first mode of vibration.

Equation (6) was used to evaluate the total displacements $u_{1}$ to $u_{4}$ at the nodal points of a segment of the vibrating beam. Though the equation represents the summation of an infinite series, an evaluation of the first few terms provides values of very good precision.

The values of $\mathrm{a}_{2 \mathrm{j}}$, and $\mathrm{a}_{4 \mathrm{j}}$ for $\mathrm{j}=1,2,3,4,5,6,7$ can be evaluated from (4) and (5). These are substituted into $(7)-(10)$ to obtain the fixed end forces, which are applied to the force equilibrium equations to get the nodal forces $P_{1}, P_{2}, P_{3}$ and $P_{4}$. And finally (17) is evaluated to obtain the stiffness modification factors $\phi_{1}, \phi_{2}, \phi_{3}$ and $\phi_{4}$.

For this beam there are two possible cases, and the method of obtaining the stiffness modification factors depends on the case being considered.

a) When $\xi_{1}$ is greater or equal to zero and $\xi_{2}$ is less than 1

In this case the segment of the fixed-pinned beam under consideration is not positioned to the far right of the beam (the end that is pinned). Hence the process of calculating the stiffness modification factors will be as outlined above.

b) When $\xi_{2}$ is equal to 1

In this case the segment under consideration is located at the far right of the fixed-pinned beam. This implies that the segment is fixed at the left end and pinned at the right end hence its stiffness matrix is different from that of (16). The proposed stiffness matrix for this beam segment is therefore

$$
\left[k_{d}\right]=\left[\begin{array}{cccc}
\frac{3 E I}{l^{3}} \phi_{1} & \frac{3 E I}{l^{2}} \phi_{2} & -\frac{3 E I}{l^{3}} \phi_{3} & 0 \\
\frac{3 E I}{l^{2}} \phi_{2} & \frac{3 E I}{l} \phi_{1} & -\frac{3 E I}{l^{2}} \phi_{4} & 0 \\
-\frac{3 E I}{l^{3}} \phi_{3} & -\frac{3 E I}{l^{2}} \phi_{4} & \frac{3 E I}{l^{3}} \phi_{1} & 0 \\
0 & 0 & 0 & 0
\end{array}\right]
$$

By substituting (18) into (15) and putting $\mathrm{P}_{4}=0$ because of the hinged end we obtain a set of three equations with four unknowns $\left(\phi_{1}\right.$ to $\left.\phi_{4}\right)$. To solve it there is need to know the value of one of the unknowns. By assuming $\phi_{4}=1$ we obtain the values of the other three as

$$
\left\{\begin{array}{l}
\phi_{1} \\
\phi_{2} \\
\phi_{3}
\end{array}\right\}=\left[\begin{array}{ccc}
\frac{3 E I}{l^{3}} u_{11} & \frac{3 E I}{l^{2}} u_{21} & -\frac{3 E I}{l^{3}} u_{31} \\
\frac{3 E I}{l} u_{21} & \frac{3 E I}{l^{2}} u_{11} & 0 \\
\frac{3 E I}{l^{3}} u_{31} & 0 & -\frac{3 E I}{l^{3}} u_{11}
\end{array}\right]^{-1}\left\{\begin{array}{c}
P_{1}+\frac{\mu\left(\xi_{2}-\xi_{1}\right)}{2} \omega^{2} u_{11} \\
P_{2}-\frac{3 E I}{l^{2}} u_{31} \\
P_{3}+\frac{\mu\left(\xi_{2}-\xi_{1}\right)}{2} \omega^{2} u_{31}+\frac{3 E I}{l^{2}} u_{21}
\end{array}\right\}
$$

Note that $\phi_{4}=1$.

Equations (17) and (19) provide a way of calculating the stiffness modification factors $\phi_{1}$ to $\phi_{4}$ for a element of a fixed-pinned beam under lateral vibration are calculated. Using these equations the values of stiffness modification factors at different values of $\xi_{1}$ and $\xi_{2}$ for the lateral vibration of 
a fixed-pinned beam can be obtained. A numerical demonstration of these steps are presented Table 1 below.

Tab 1 - Calculation of the Stiffness modification factor for an element positioned at $\xi_{1}=0, \xi_{2}=0.5$ on a propped cantilever beam under lateral vibration

\begin{tabular}{|c|c|}
\hline & $\xi_{1}=0, \quad \xi_{2}=0.5$ \\
\hline From Equation (6) & \\
\hline$u_{11}=0$ & $u_{31}=-0.2500280951625$ \\
\hline $\mathrm{u}_{21}=0$ & $u_{41}=-0.2501752736690$ \\
\hline From Equations (7) to (10) & \\
\hline$F_{1}=8.98435096276653$ & $F_{3}=11.92853399194589$ \\
\hline$F_{2}=0.92592074058035$ & $F_{4}=-0.98636146391392$ \\
\hline From force equilibrium equ & uations (see Figure 3) \\
\hline$P_{1}=26.98284153031153$ & $P_{3}=-6.06995657559912$ \\
\hline$P_{2}=5.92589392980464$ & $P_{4}=3.01291063063430$ \\
\hline From equation (17) taking & $E I=1$ \\
\hline$\phi_{1}=1.11502214241540$ & $\phi_{3}=1.45734448442994$ \\
\hline$\phi_{2}=0.90229998347702$ & $\phi_{4}=1.24678725977081$ \\
\hline
\end{tabular}

A sample Matlab program for the calculation of the stiffness modification factors can also be written.

\section{RESULTS}

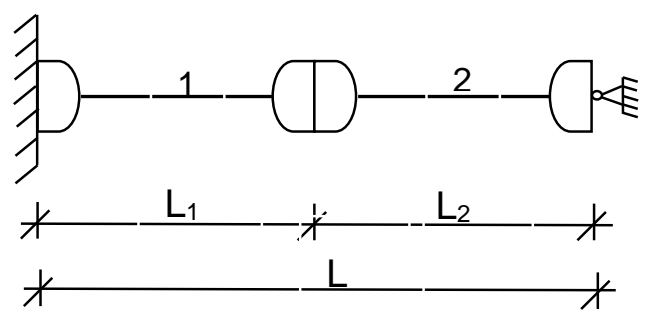

(a)

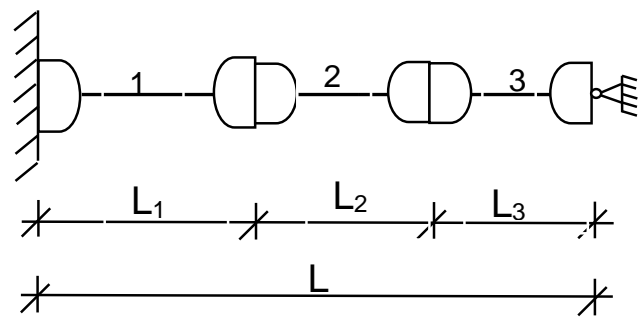

(b)

Fig. 4 - Some lumped mass propped cantilever beams

For the propped cantilever beam of Figure $4 a$, the lumped mass is at a distance $L_{1}$ from the fixed end. By solving it for different values of $L_{1}$ using the steps outlined in Table 1 and comparing results with that from the finite element model we obtain the results presented in Table 2 . For the finite element model the inertia matrix used was the popular consistency matrix derived from the shape functions.

Tab 2 - Natural frequency of a lumped mass propped cantilever of Figure 4a 
Article no. 62

CIVIL

ENGINEERING

JOURNAL

THE CIVIL ENGINEERING JOURNAL 4-2021

\begin{tabular}{|l|l|l|l|l|l|}
\hline \multirow{2}{*}{$S / N$} & \multirow{2}{*}{$L_{1} / \mathrm{L}$} & \multirow{2}{*}{$\mathrm{L}_{2} / \mathrm{L}$} & \multicolumn{4}{|c|}{ Natural frequency $\omega$ in $\sqrt{E I / \mu L^{4}}$} \\
\cline { 4 - 6 } & & & Lagrange without $\phi$ & \multicolumn{1}{|c|}{ Lagrange with $\phi$} & Finite Element Model \\
\hline 1 & $1 / 10$ & $9 / 10$ & 87.1627 & 15.4182 & 373.5870 \\
\hline 2 & $2 / 10$ & $8 / 10$ & 35.1220 & 15.4182 & 93.3968 \\
\hline 3 & $3 / 10$ & $7 / 10$ & 22.1424 & 15.4182 & 41.5097 \\
\hline 4 & $4 / 10$ & $6 / 10$ & 17.0103 & 15.4182 & 23.3492 \\
\hline 5 & $5 / 10$ & $5 / 10$ & 14.8131 & 15.4182 & 14.9435 \\
\hline 6 & $6 / 10$ & $4 / 10$ & 14.2915 & 15.4182 & 10.3774 \\
\hline 7 & $7 / 10$ & $3 / 10$ & 15.3490 & 15.4182 & 7.6242 \\
\hline 8 & $8 / 10$ & $2 / 10$ & 19.1366 & 15.4182 & 5.8373 \\
\hline 9 & $9 / 10$ & $1 / 10$ & 35.582 & 15.4182 & 4.6122 \\
\hline
\end{tabular}

From the results in Table 2 we observe that the values of the natural frequency obtained from the lumped mass beam varied with the relative values of $L_{1}$ and $L_{2}$. It varied more with increase in the difference between $L_{1}$ and $L_{2}$ and gave the best prediction of the fundamental frequency when $L_{1}$ was equal to $L_{2}$. The results in Table 2 also shows that using the Lagrange's equations it is possible to obtain a near accurate value of natural frequency by a careful section of the relative values of $L_{1}$ and $L_{2}$. For instance at $L_{1} / L=0.7$ the value of natural frequency obtained was $15.35 \mathrm{~Hz}$ which has an error of only $0.45 \%$. This is not so with the finite element at the best value is only available when $L_{1}=L_{2}$. However with the application of the stiffness modification factors the obtained values of fundamental natural frequency was exact irrespective of the relative values of $L_{1}$ and $L_{2}$.

The natural frequency values obtained from a finite element model continued to decrease steadily with an increase in the ratio of $L_{1}$ to $L_{2}$. It gave the maximum value at $L_{1} / L=1 / 10, L_{2} / L=$ $9 / 10$. The trend is better appreciated in the plot of natural frequency against $L_{1} / L$ presented in Fig 5 .

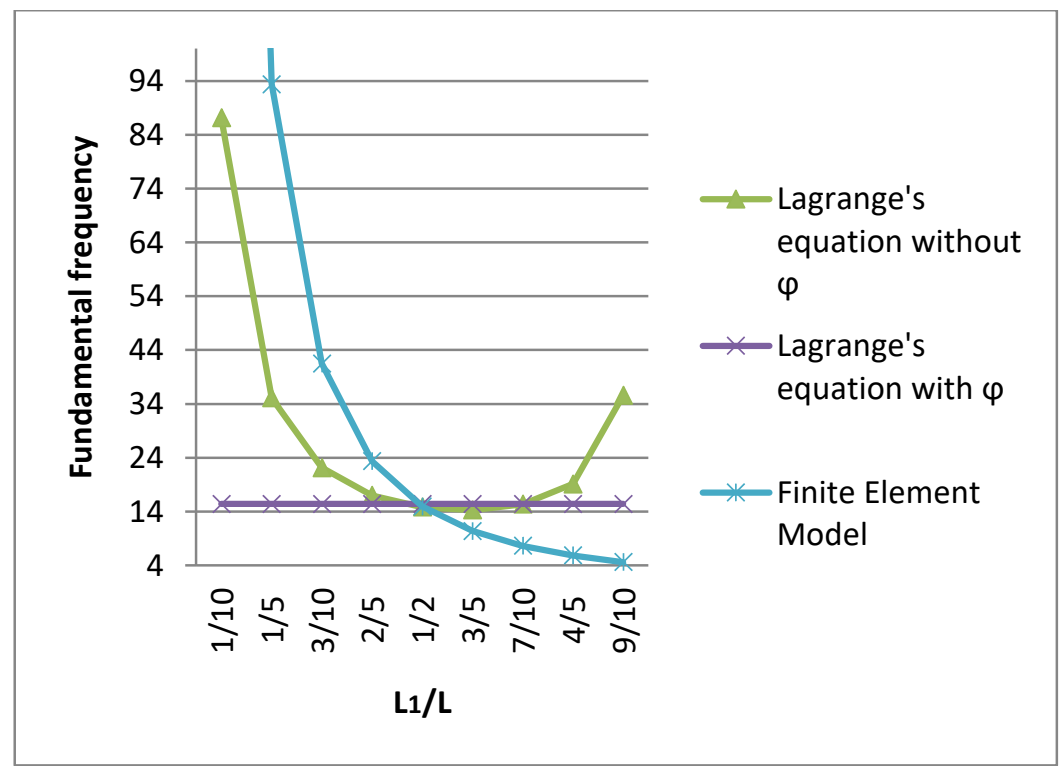

Fig. 5 - Fundamental frequencies as functions of $L_{1} / L$ for the propped cantilever beam presented in Fig. $4 a$

From Figure 5 it is observed that the plots for analysis with and without stiffness modification factors and finite element model tend to converge at $L_{1} / L=0.5$. At this point $L_{1}=L_{2}$. It shows that 


\section{CIVIL}

ENGINEERING

JOURNAL

analysis with Lagrange's equation without $\phi$ gives its best results when the lumps are evenly spaced. This is also true of the finite element model as its best result of $14.94 \mathrm{~Hz}$ with an error of $3.1 \%$ was obtained when $L_{1}$ was equal to $L_{2}$. The error margins increased continuously as the difference between $L_{1}$ and $L_{2}$ increased. The plot of natural frequencies obtained with the application of the stiffness modification was a horizontal line showing that it was not affected by the relative values of $\mathrm{L}_{1}$ and $\mathrm{L}_{2}$.

To further study the effect of mass lumping and the stiffness modification factors on the accuracy of results the beam of Figure $4 \mathrm{~b}$ was analyzed using the Lagrange's equation and the finite element model. The result is presented in Table 3.

Tab. 3 - Natural frequency of a lumped mass propped cantilever of Fig. $4 b$

\begin{tabular}{|c|c|c|c|c|c|c|}
\hline \multirow[b]{2}{*}{$\mathrm{S} / \mathrm{N}$} & \multirow[b]{2}{*}{$L_{1}$} & \multirow[b]{2}{*}{$\mathrm{L}_{2}$} & \multirow[b]{2}{*}{$\mathrm{L}_{3}$} & \multicolumn{3}{|c|}{ Natural frequency $\omega$ in $\sqrt{E I / \mu L^{4}}$} \\
\hline & & & & $\begin{array}{l}\text { Lagrange without } \\
\phi\end{array}$ & Lagrange with $\phi$ & $\begin{array}{l}\text { Finite Element } \\
\text { Model (FEM) }\end{array}$ \\
\hline 1 & $1 / 12$ & $1 / 12$ & $10 / 12$ & 45.6106 & 15.4182 & 245.1372 \\
\hline 2 & $1 / 12$ & $2 / 12$ & $9 / 12$ & 28.0664 & 15.4182 & 87.5877 \\
\hline 3 & $1 / 12$ & $3 / 12$ & $8 / 12$ & 20.7803 & 15.4182 & 44.4924 \\
\hline 4 & $1 / 12$ & $4 / 12$ & $7 / 12$ & 17.2032 & 15.4182 & 26.8425 \\
\hline 5 & $1 / 12$ & $5 / 12$ & $6 / 12$ & 15.4543 & 15.4182 & 17.9366 \\
\hline 6 & $1 / 12$ & $6 / 12$ & $5 / 12$ & 14.8974 & 15.4182 & 12.8258 \\
\hline 7 & $1 / 12$ & $7 / 12$ & $4 / 12$ & 15.4312 & 15.4182 & 9.6244 \\
\hline 8 & $1 / 12$ & $8 / 12$ & $3 / 12$ & 17.4598 & 15.4182 & 7.4876 \\
\hline 9 & $1 / 12$ & $9 / 12$ & $2 / 12$ & 22.6398 & 15.4182 & 5.9907 \\
\hline 10 & $1 / 12$ & $10 / 12$ & $1 / 12$ & 39.6405 & 15.4182 & 4.9016 \\
\hline 11 & $2 / 12$ & $1 / 12$ & $9 / 12$ & 28.1887 & 15.4182 & 138.8296 \\
\hline 12 & $2 / 12$ & $2 / 12$ & $8 / 12$ & 21.2239 & 15.4182 & 61.2843 \\
\hline 13 & $2 / 12$ & $3 / 12$ & $7 / 12$ & 17.7105 & 15.4182 & 34.3255 \\
\hline 14 & $2 / 12$ & $4 / 12$ & $6 / 12$ & 15.9719 & 15.4182 & 21.8969 \\
\hline 15 & $2 / 12$ & $5 / 12$ & $5 / 12$ & 15.4212 & 15.4182 & 15.1687 \\
\hline 16 & $2 / 12$ & $6 / 12$ & $4 / 12$ & 15.9704 & 15.4182 & 11.1231 \\
\hline 17 & $2 / 12$ & $7 / 12$ & $3 / 12$ & 18.0181 & 15.4182 & 8.5034 \\
\hline 18 & $2 / 12$ & $8 / 12$ & $2 / 12$ & 23.1101 & 15.4182 & 6.7106 \\
\hline 19 & $2 / 12$ & $9 / 12$ & $1 / 12$ & 36.8379 & 15.4182 & 5.4303 \\
\hline 20 & $3 / 12$ & $1 / 12$ & $8 / 12$ & 20.7848 & 15.4182 & 89.5397 \\
\hline 21 & $3 / 12$ & $2 / 12$ & $7 / 12$ & 17.6069 & 15.4182 & 45.1938 \\
\hline 22 & $3 / 12$ & $3 / 12$ & $6 / 12$ & 16.0110 & 15.4182 & 27.2375 \\
\hline 23 & $3 / 12$ & $4 / 12$ & $5 / 12$ & 15.5023 & 15.4182 & 18.1792 \\
\hline 24 & $3 / 12$ & $5 / 12$ & $4 / 12$ & 16.0011 & 15.4182 & 12.9836 \\
\hline 25 & $3 / 12$ & $6 / 12$ & $3 / 12$ & 17.7862 & 15.4182 & 9.7320 \\
\hline 26 & $3 / 12$ & $7 / 12$ & $2 / 12$ & 21.5862 & 15.4182 & 7.5638 \\
\hline
\end{tabular}


Article no. 62

CIVIL

ENGINEERING

JOURNAL

THE CIVIL ENGINEERING JOURNAL 4-2021

\begin{tabular}{|c|c|c|c|c|c|c|}
\hline 27 & $3 / 12$ & $8 / 12$ & $1 / 12$ & 26.4053 & 15.4182 & 6.0465 \\
\hline 28 & $4 / 12$ & $1 / 12$ & $7 / 12$ & 17.0131 & 15.4182 & 62.8185 \\
\hline 29 & $4 / 12$ & $2 / 12$ & $6 / 12$ & 15.5421 & 15.4182 & 34.7074 \\
\hline 30 & $4 / 12$ & $3 / 12$ & $5 / 12$ & 15.0313 & 15.4182 & 22.1212 \\
\hline 31 & $4 / 12$ & $4 / 12$ & $4 / 12$ & 15.3490 & 15.4182 & 15.3211 \\
\hline 32 & $4 / 12$ & $5 / 12$ & $3 / 12$ & 16.5433 & 15.4182 & 11.2307 \\
\hline 33 & $4 / 12$ & $6 / 12$ & $2 / 12$ & 18.5256 & 15.4182 & 8.5814 \\
\hline 34 & $4 / 12$ & $7 / 12$ & $1 / 12$ & 20.0733 & 15.4182 & 6.7686 \\
\hline 35 & $5 / 12$ & $1 / 12$ & $6 / 12$ & 15.0016 & 15.4182 & 46.6862 \\
\hline 36 & $5 / 12$ & $2 / 12$ & $5 / 12$ & 14.4051 & 15.4182 & 27.5194 \\
\hline 37 & $5 / 12$ & $3 / 12$ & $4 / 12$ & 14.4924 & 15.4182 & 18.3201 \\
\hline 38 & $5 / 12$ & $4 / 12$ & $3 / 12$ & 15.1630 & 15.4182 & 13.0819 \\
\hline 39 & $5 / 12$ & $5 / 12$ & $2 / 12$ & 16.1582 & 15.4182 & 9.8055 \\
\hline 40 & $5 / 12$ & $6 / 12$ & $1 / 12$ & 16.7747 & 15.4182 & 7.6200 \\
\hline 41 & $6 / 12$ & $1 / 12$ & $5 / 12$ & 14.0630 & 15.4182 & 36.1680 \\
\hline 42 & $6 / 12$ & $2 / 12$ & $4 / 12$ & 13.9468 & 15.4182 & 22.3849 \\
\hline 43 & $6 / 12$ & $3 / 12$ & $3 / 12$ & 14.2934 & 15.4182 & 15.4255 \\
\hline 44 & $6 / 12$ & $4 / 12$ & $2 / 12$ & 14.8462 & 15.4182 & 11.2985 \\
\hline 45 & $6 / 12$ & $5 / 12$ & $1 / 12$ & 15.1337 & 15.4182 & 8.6329 \\
\hline 46 & $7 / 12$ & $1 / 12$ & $4 / 12$ & 16.5351 & 15.4182 & 28.9079 \\
\hline 47 & $7 / 12$ & $2 / 12$ & $3 / 12$ & 16.6705 & 15.4182 & 18.5901 \\
\hline 48 & $7 / 12$ & $3 / 12$ & $2 / 12$ & 17.1969 & 15.4182 & 13.1734 \\
\hline 49 & $7 / 12$ & $4 / 12$ & $1 / 12$ & 17.5268 & 15.4182 & 9.8571 \\
\hline 50 & $8 / 12$ & $1 / 12$ & $3 / 12$ & 14.7732 & 15.4182 & 23.6730 \\
\hline 51 & $8 / 12$ & $2 / 12$ & $2 / 12$ & 15.0215 & 15.4182 & 15.7046 \\
\hline 52 & $8 / 12$ & $3 / 12$ & $1 / 12$ & 15.1414 & 15.4182 & 11.3879 \\
\hline 53 & $9 / 12$ & $1 / 12$ & $2 / 12$ & 16.9415 & 15.4182 & 19.7664 \\
\hline 54 & $9 / 12$ & $2 / 12$ & $1 / 12$ & 17.1041 & 15.4182 & 13.4574 \\
\hline 55 & $10 / 12$ & $1 / 12$ & $1 / 12$ & 22.0751 & 15.4182 & 16.7688 \\
\hline
\end{tabular}

To produce the results of Table 3 , the lumped mass of Figure $4 \mathrm{~b}$ were moved at $\mathrm{L} / 12$ and the fundamental natural frequency calculated at all possible positions of the lumped masses. The results in the Table show that the calculated natural frequency varied with the relative values of $L_{1}, L_{2}$ and $L_{3}$. The same was observed in frequency values obtained from a finite element analyses. To appreciate the trend in variation of the obtained natural frequency with the spacing of the lumped mass we present an interaction plot showing the variation of frequency with the values of $L_{1} / L$ and $\mathrm{L}_{2} / \mathrm{L}$ in Figure 6. 


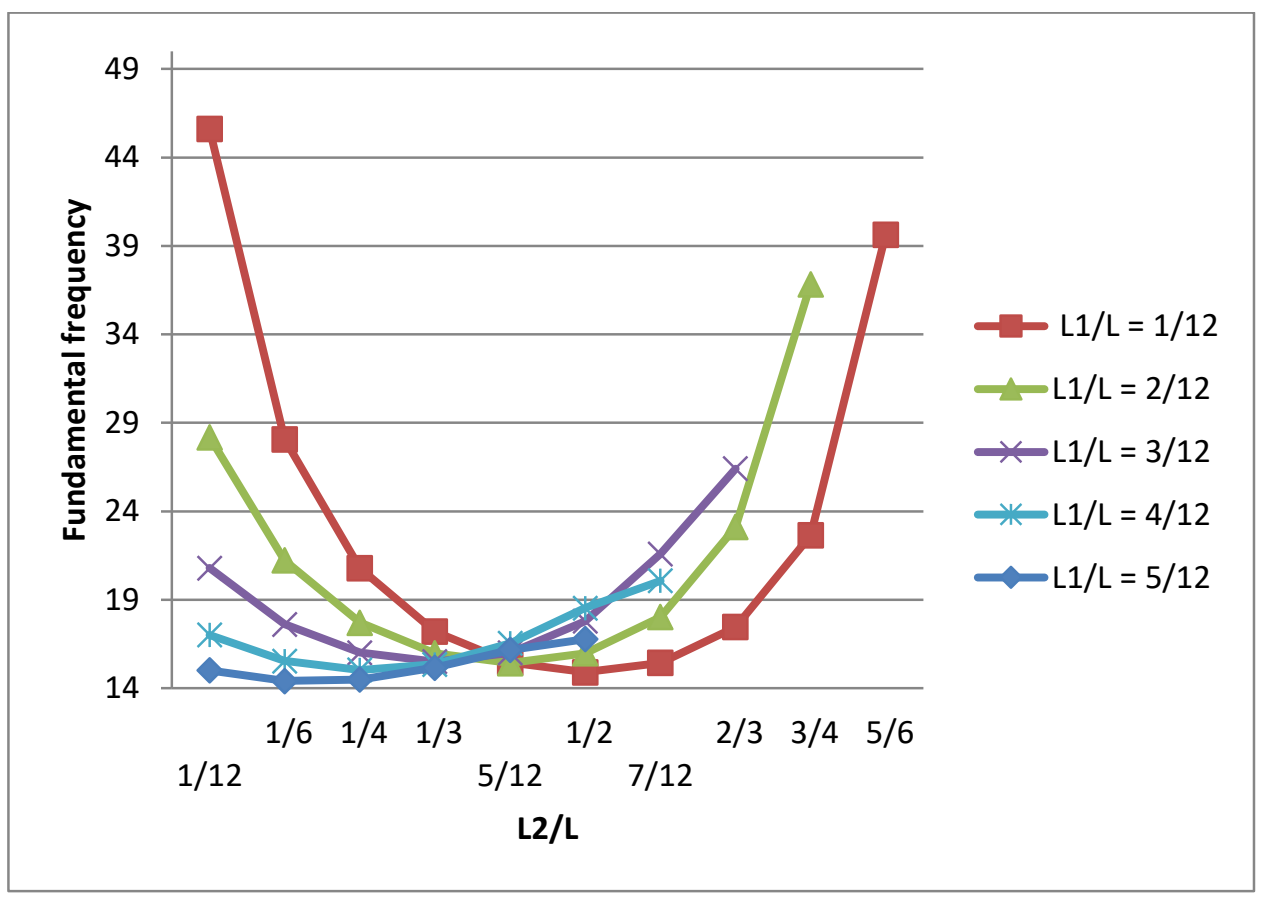

Fig. 6 - Interaction plots of fundamental frequency against $L_{2} / L$ at different values of $L_{1} / L$ using the Lagrange's equations

Figure 6 shows the interaction plots for the $L_{1} / L$ and $L_{2} / L$ when using Lagrange's equation on the lumped masses. In Figure 6 only the values of $L_{1}$ and $L_{2}$ were considered because if we know the values of $L_{1}$ and $L_{2}$ the values of $L_{3}$ becomes defined and can be obtained from subtracting $L_{1}$ and $L_{2}$ from the total length of the beam. We first observe that each of the curves tend to have a $U$ shape. This shows that for almost all values of $L_{1} / L$ there are for each two possible values of $L_{2} / L$ that will give a fundamental frequency value of the beam to a good precision. The curves are not parallel and this shows that there is some level of interaction between the values of $L_{1}$ and $L_{2}$ on the natural frequency values obtained. The curves all appear to converge between the values of $L_{2} / L=$ $1 / 3$ and $L_{2} / L=5 / 12$. At this value the value of $L_{1} / L$ tend to have minimal effects on the calculated natural frequency. 


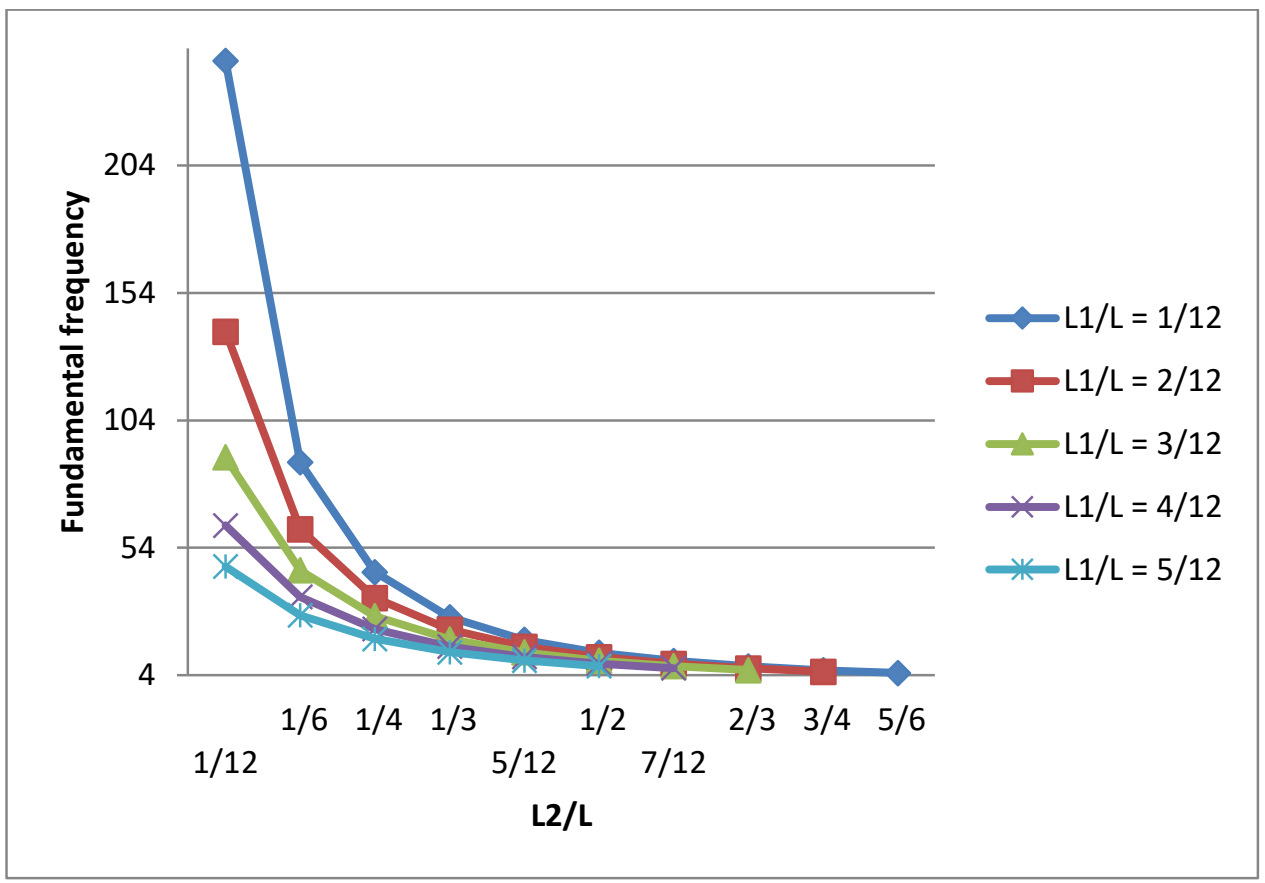

Fig. 7 - Interaction plots of calculated fundamental frequency against $L_{2} / L$ at different values of $L_{1} / L$ using the finite element model

Unlike that obtained in Figure 6 the plots in Figure 7 are like exponential plots. The values of calculated frequency decreases with increases in the values of $L_{2} / L$ at constant $L_{1} / L$. Likewise the values of calculated frequency also decreases with increase in the values of $L_{1} / L$ at constant $L_{2} / L$. For every value of $L_{1} / L$ there is one possible value of $L_{2} / L$ that will give a good approximation of the exact fundamental frequency. By zooming into the graph (see Figure 8) we observe that the lines of equal values of $L_{1} / L$ are near parallel in the neighborhood of $\omega=15 \mathrm{~Hz}$.

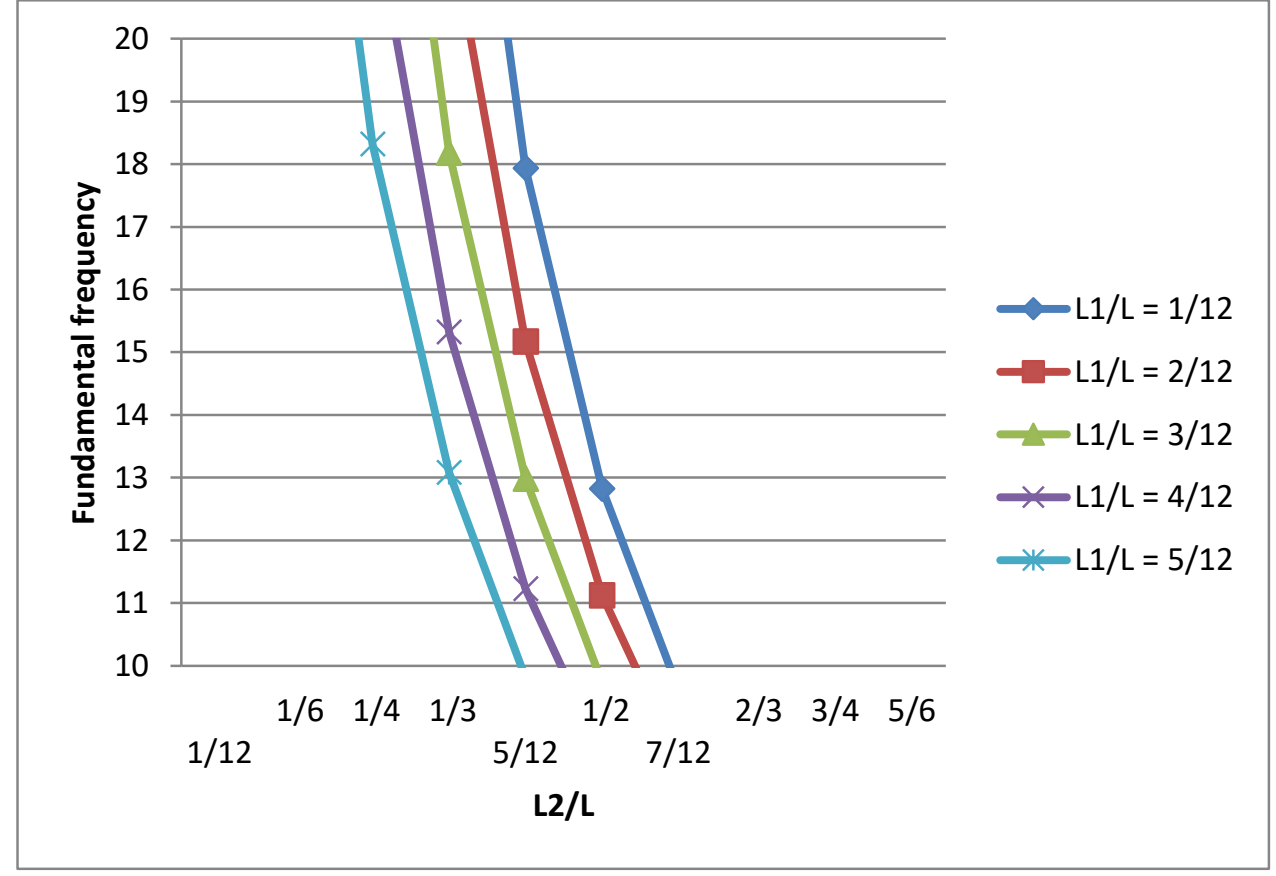

Fig. 8 - Enlarged Interaction plots of calculated fundamental frequency against $L_{2} / L$ at different values of $L_{1} / L$ using the finite element model 
From Figure 8 it would be observed that there seems to be not interaction between $L_{1} / L$ and $L_{2} / L$ between values of $L_{2} / L=1 / 3$ and $1 / 2$. At these values any change in the values of $L_{1} / L$ does not have significant effects on the calculated values of the fundamental frequency. This is evident in the near parallel nature of the lines of points of equal $L_{1} / L$ in Figure 8.

From the Tables 2 and 3 it would be observed that the natural frequencies obtained from the use of Lagrange's equations on the propped cantilever that had its mass lumped had some measure of errors. When the stiffness of the system was however modified using the stiffness modification factors, the use of Lagrange's equations was able to predict accurately the fundamental frequencies irrespective of the position of lumped mass.

\section{CONCLUSION}

This work improved the values of fundamental frequencies obtained in the analysis of continuous systems as having discrete masses connected by mass-less elements using a propped cantilever as a case study. Even though the stiffness modification factors is a product of some rigorous mathematical manipulation, its implementation is largely simplified by the use of Matlab software. Hence the calculation of the stiffness modification can be automated. From this work we can infer that

1) To obtain an accurate dynamic response from a lumped mass beam there may be need to modify the stiffness composition/distribution of the system.

2) There is no linear modification of the stiffness distribution of a lumped mass beam under lateral vibration that can cause it to be dynamically equivalent to the continuous beam. This is so because the values of $\phi_{1}, \phi_{2}, \phi_{3}$ and $\phi_{4}$ obtained for each segment as shown in Table 1 are not equal.

3) A careful selection of the relative positions of the lumped masses can lead to results with very good accuracy.

4) Having more lumps will lead to a better results just as breaking a structure into more elements in finite element analysis will give a better result.

This work lays the foundations for precise analysis of structures by lumping its distributed mass at select nodes. The mass lumping is an idealization that simplifies the analysis of the structure while the stiffness modification factors helps in keeping the results of the analysis accurate.

In the formulation of the equations of end forces only the deformation due to bending moments were considered. The effects of shearing and axial stresses on deformation were ignored in order to simplify the analysis. Further work including their effects is recommended.

\section{ACKNOWLEDGEMENTS}

We want to specially thank Late Prof I. O. Onyeyili, the former deputy vice-chancellor of Nnamdi Azikiwe University for his technical support to us in carrying out this research

\section{REFERENCES}

[1] A. A. Shabana, Vibration of Continuous Systems. In:Theory of Vibration, Mechanical Engineering Series, Springer, New York, NY pp 175-251, 1991

[2] W. T. Thomson and M. D. Dahleh, Theory of Vibrations with Applications. $5^{\text {th }}$ Edition, Prentice Hall New Jersey, 1998

[3] S. Rajasekaran, Structural Dynamics of Eathquake Engineering: Theory and Application using Mathematica and Matlab, Woodhead Publishing Limited Cambridge, 2009

[4] J. Humar, Dynamics of structures, $3^{\text {rd }}$ Edition, CRC Press, London, 2012 
[5] M. Simsek, "Vibration analysis of a functionally graded beam under a moving mass by using different beam theories", Composite Structures vol. 92, no. 4, pp 904-917, Elsevier, 2010, https://doi.org/10.1016/i.compstruct.2009.09.030

[6] Chung J., Yoo H. H., "Dynamic Analysis of a rotating cantilever beam by using the finite element method", Journal of Sound and Vibration,vol. 249, Issue 1, pp 147-164, Elsevier, 2002, https://doi.org/10.1006/jsvi.2001.3856

[7] L. Li, Y. Hu, X.Li, "Longitudinal vibration of size dependent rods via non-local strain gradient theory", International Journal of Mechanical Science, vols. 115-116, pp 135-144, Elsevier, 2016, https://doi.org/10.1016/j.ijmecsci.2016.06.011

[8] Y. Saad and H. A. V. Vorst, "Iterative Solution of Linear Systems in the 20 th Century", Journal of Computational and Applied Mathematics Vol 123, Issue 1-2 pp 1-33, 2000, https://doi.org/10.1016/S0377-0427(00)00412-X

[9] N. J. Fergusson, W. D. Pilkey, "Frequency-dependent element matrices", Journal of Applied Matrices, vol.59. issue 1, pp 1-10, 1992, https://doi.org/10.1115/1.2899418

[10] Downs B., "Vibration Analysis of Continuous systems by Dynamic discretization", Journal of Mechanical Design, vol. 102, Issue 2. 1980. https://doi.org/10.1115/1.3254757

[11] A. Houmat, Nonlinear free vibration of a shear deformable laminated composite annular elliptical plate, ActaMechanica. Springer 208:281. https://doi.org/10.1007/s00707-009-0148-5

[12] P. Beaurepaire and G. I. Schueller, "Modelling of the Variability of fatigue Crack growth using cohesive zone element", Engineering Fracture Mechanics vol. 78 Issue 12 pp 2399-2413 Elsevier. 2011. https://doi.org/10.1016/i.engfracmech.2011.05.011

[13] F. Tornabene, F. Nicholas, F. Uberlini, V. Erasmo, "Strong Formulation Finite Element Method based on Differential Quadrature: A Survey", Applied Mechanics Review vol. 67 pp 1-50 ASME 2015. https://doi.org/10.1115/1.4028859

[14] G. Cocchetti, M. Pagani, U. Perego, "Selective mass scaling for distorted solid -shell elements in explicit dynamics:Optimal scaling factor and stable time step estimate", International Journal for Numerical Methods in Engineering, vol. 101, no. 9, pp 1-30 2013. https://doi.org/10.1002/nme.4829

[15] X. Hua, T. C. Lin, T. Peng, W. E. Wali, "Dynamic Analysis of spiral level geared rotor systems applying finite elements and enhanced lumped parameters", International Journal of Automotive Technology,

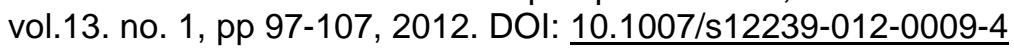

[16] G. Kouroussis, G. Gazetas, I. Anastasopoulos, C. Conti, O. Verlinden, "Lumped mass model of vertical dynamic coupling of a railway track on elastic homogenous or layered halfspace", Proceedings of the $8^{\text {th }}$ International Conference on Structural Dynamics EURODYN Belgium, pp 676-683, 2011

[17] M. Blundell, D. Harty, The multibody systems approach to vehicle dynamics, Butterworth-Heinemann Uk, pp 185-334, 2015

[18] G. Kouroussis, O. Verlinden, "Prediction of railway ground vibrations: Accuracy of a coupling lumped mass model for representing the track/soil interaction", Soil Dynamics and Earthquake Engineering, vol. 69, pp. 220-226, Elsevier, 2015. https://doi.org/10.1016/j.soildyn.2014.11.007

[19] K. K. Reichl, D. J. Inman, Lumped mass model of a 1D metastructure for vibration suppression with no additional mass, Journal of Sound and Vibration, vol. 403, pp. 75-89, Elsevier, 2017. https://doi.org/10.1016/j.jsv.2017.05.026

[20] H. Ahmadian, M. I. Friswell, J. E. Mottershead, "Minimization of the discretization error in mass and stiffness formulations by an inverse method". International Journal for Numerical Methods in Engineering, vol. 41, no. 2, pp 371-387, 1998. https://doi.org/10.1002/(SICl)10970207(19980130)41:2<371::AID-NME288>3.0.CO;2-

[21] C. Hager, B. I. Wohlmuth, "Analysis of a space-time discretization for dynamic elasticity problems based on mass-free surface elements", SIAM Journal on Numerical Analysis, vol. 47, no. 3, pp 1863-1885, 2009. https://doi.org/10.1137/080715627

[22] D. Wang, W. Liu, H. Zhang, "Superconvergent Isogeometric free vibration analysis of Euler-Bernoulli Beams and Kirchhoff plates with higher order mass matrices", Computer Methods in Applied Mechanics and Engineering, vol. 286, pp. 230-267, 2015. https://doi.org/10.1016/i.cma.2014.12.026

[23] T. M. Ericson, R. G. Parker, "Planetary gear modal vibration experiments and correlation against lumped-parameter and finite element models", Journal of Sound and Vibration, vol. 332, no.9, pp 23502375 Elservier, 2013. https://doi.org/10.1016/j.jsv.2012.11.004 
[24] I. O. Onyeyili, C. H. Aginam, V. O. Okonkwo, "Analysis of a propped cantilever under longitudinal vibration by a modification of the system's stiffness distribution", IOSR Journal of Mechanical and Civil Engineering, vol. 13, Iss. 5, pp 65-78, 2016

[25] T. R. Tauchert, 1974. Energy Principles in Structural Mechanics. International Student Edition, McGraw-Hill Kogakusha Ltd Tokyo, 1974

[26] J. Buskiewicz, "A Dynamic Analysis of a coupled beam/slider system", Applied Mathematical Modelling vol. 32, Issue 10, pp 1941 - 1955, 2008. https://doi.org/10.1016/j.apm.2007.06.028

[27] V. O. Okonkwo, Dynamic Analysis of frames by the modification of the system's stiffness distribution, Unpublished PhD Thesis, Civil Eng. NnamdiAzikwe University, Awka

[28] V. O. Okonkwo, Analysis of Multi-storey steel frames, Unpublished MEng Thesis, Civil Eng. Nnamdi Azikwe University, Awka

[29] R. L. Bisplinghoff, H. Ashley, R. L. Halman, Aeroelasticity, Cambridge Mass Addison-Wesley Publishing Company Inc. pp 90, 1955 